\title{
Crosstalk between human endometrial stromal cells and decidual NK cells promotes decidualization in vitro by upregulating IL-25
}

\author{
YUAN ZHANG $^{1 *}$, YING WANG $^{2 *}$, XIAO-HUI WANG ${ }^{3}$, WEN-JIE ZHOU ${ }^{1}$, LI-PING JIN ${ }^{1,3}$ and MING-QING LI ${ }^{1}$ \\ ${ }^{1}$ Laboratory for Reproductive Immunology, Hospital of Obstetrics and Gynecology, \\ Fudan University, Shanghai Medical College, Shanghai 200011; ${ }^{2}$ Department of Obstetrics and Gynecology, \\ Qilu Hospital of Shandong University, Jinan, Shandong 250012; ${ }^{3}$ Clinical and Translational Research Center, \\ Shanghai First Maternity and Infant Hospital, Tongji University School of Medicine, Shanghai 201204, P.R. China
}

Received August 21, 2017; Accepted October 10, 2017

DOI: $10.3892 / \mathrm{mmr} .2017 .8267$

\begin{abstract}
Embryo implantation is essential for a successful pregnancy, and leads to the decidualization of endometrial stromal cells (ESCs) in the secretory phase of the menstrual cycle. It has previously been demonstrated that decidual stromal cells (DSCs) co-express interleukin (IL)-25/IL-17RB and that IL-25 further promotes the proliferation of DSCs via activating c-Jun $n$-terminal kinase and protein kinase $B$ signals, therefore the present study primarily focused on the role of IL-25 in the process of decidualization in vitro. It was demonstrated that the expression of IL-25/IL-17RB in ESCs was decreased compared with DSCs. In addition, following decidualization, the expression levels of IL-25/IL-17RB in ESCs were significantly elevated. Recombinant human (rh) IL-25 promoted the decidualization of ESCs in the presence of 8-bromoadenosine 3',5'-cyclic monophosphate sodium salt and $6 \alpha$-methyl17 $\alpha$-acetoxyprogesterone, which was partially inhibited by anti-human IL-25 neutralizing antibody (anti-IL-25) or anti-IL-17RB. In addition, decidual natural killer (dNK) cells not only secreted IL-25, however also further accelerated the decidualization in vitro. Therefore, these findings indicated that ESCs differentiate into DSCs in the presence of ovarian hormones, resulting in the upregulation of IL-25/IL-17RB
\end{abstract}

Correspondence to: Dr Ming-Qing Li, Laboratory for Reproductive Immunology, Hospital of Obstetrics and Gynecology, Fudan University, Shanghai Medical College, 413 Zhao Zhou Road, Shanghai 200011, P.R. China

E-mail: mqli@fudan.edu.cn

Professor Li-Ping Jin, Clinical and Translational Research Center, Shanghai First Maternity and Infant Hospital, Tongji University School of Medicine, 2699 West Gaoke Road, Shanghai 201204, P.R. China

E-mail: jinlp01@163.com

${ }^{*}$ Contributed equally

Key words: interleukin-25, interleukin-17RB, endometrial stromal cells, decidualization, decidual natural killer cells expression in ESCs. Furthermore, IL-25 secreted by ESCs and $\mathrm{dNK}$ cells further facilitates the decidualization of ESCs, which may form a positive feedback mechanism at the maternal-fetal interface and thus contribute to the establishment and maintenance of normal pregnancy.

\section{Introduction}

Embryo implantation is important for a successful pregnancy, and this depends on the bidirectional crosstalk between a vital embryo and a receptive endometrium (1). This triggers a series of responses to ovarian steroid hormones, collectively termed decidualization, during which the elongated fibroblast-like endometrial stromal cells (ESCs) undergo proliferation and differentiation into larger, spherical decidual cells (2-5). At the same time, immune cells of the uterus also undergo consequential alterations, including an influx of macrophages and proliferation of uterine natural killer (NK) cells (6), which contribute $30-40 \%$ of the leukocytes in the decidua during the first trimester. Inappropriate implantation and placentation lead to infertility $(7,8)$ and are thought to result in other obstetric complications $(9,10)$.

In the process of decidualization, a lot of genes are upregulated or downregulated in ESCs (11), including prolactin (PRL) and insulin growth factor binding protein 1 (IGFBP-1) which increase, and are thus thought to be specific markers of decidualization (12). Previous studies have revealed that progesterone combined with other endocrine factors, including prostaglandin E2, relaxin, gonadotrophins, and cyclic adenosine monophosphate (cAMP), may trigger the process $(12,13)$. The combination of 8 -bromoadenosine 3',5'-cyclic monophosphate sodium salt (8-br-cAMP) and $6 \alpha$-methyl17 $\alpha$-acetoxyprogesterone (MPA) stimulate the decidualization effectively (12).

IL-25, additionally termed IL-17E, is a member of the IL-17 family, which includes IL-17A-F. Unlike the role of other members of the IL-17 family, IL-25 appears to be involved in the promotion of the $\mathrm{T}$ helper (Th) 2 cell immune response in allergies, asthma, and enteric nematode infections (14). IL-17RB, the principal recognized IL-25 receptor, is a $56-\mathrm{kDa}$ single transmembrane protein, which has a substantial effect on various cells by interacting with IL-25, including eosinophils, mast cells, 
monocytes and T cells. Previous studies have demonstrated that IL-25 enhances human umbilical vein endothelial cell proliferation and the length, number, and area of microvessel structures in a concentration-dependent manner in vitro, and additionally promotes angiogenesis $(15,16)$. The downregulation of IL-25 in villus from patients with recurrent miscarriages may alter the Th1/Th2 ratio (17), suggesting that IL-25 may have an important role in maintaining a successful pregnancy.

The authors previously demonstrated that human chorionic gonadotrophin (hCG) derived from trophoblasts upregulates the expression of IL-25/IL-17RB in decidual stromal cells (DSCs) and that IL-25 further stimulates the proliferation of DSCs by activating c-Jun n-terminal kinase (JNK) and protein kinase B (AKT) signaling pathways, contributing to the establishment and maintenance of normal pregnancy (18). However, when the expression of IL-25 or the concentration of hCG is abnormal, it may influence the proliferation of DSCs and result in abortion. Regarding the differentiation of ESCs into DSCs, whether ESCs express IL-25 and if IL-25 exhibits a role in the decidualization process, remains to be elucidated. Therefore, the present study aimed to investigate the expression of IL-25 in ESCs and its function in decidualization.

\section{Materials and methods}

Specimen collection. All tissue samples were collected with informed consent according to the requirements of the Research Ethics Committee in the Obstetrics and Gynecology Institute, Fudan University Shanghai Medical College (Shanghai, China). All subjects completed informed consent forms for collection of tissue samples. In addition, the present study was specifically approved by the Research Ethics Committee in the Obstetrics and Gynecology Institute, Fudan University, Shanghai Medical College (Shanghai, China). Endometrial samples $(n=18)$ were obtained from healthy women with a regular menstrual cycle (age 25-45 years). The women had not taken medications or received hormonal therapy for at least 6 months prior to surgery, nor did they have medical histories, including high blood pressure, diabetes, infertility, or spontaneous abortion. The curettage procedure was performed during the secretory phase of the menstrual cycle. In addition, pre-operative examination demonstrated no pathogen infection, and the endometrium was normal (verified by histopathological studies). Decidual samples $(n=18)$ were obtained from normal pregnant women (age 29.24 \pm 3.17 years; gestational age $8.11 \pm 1.37$ weeks; mean \pm standard deviation) whose pregnancies were terminated for non-medical reasons.

Cell isolation and culture. The endometrial tissues were immediately placed into cold Dulbecco's modified Eagle's medium (DMEM; Invitrogen; Thermo Fisher Scientific, Inc., Waltham, MA, USA), transported to the laboratory within $1 \mathrm{~h}$ following surgery, and washed with Hank's balanced salt solution for isolation of ESCs. The ESCs were isolated as previously described (19), and the purity of ESCs was approximately $90 \%$. ESCs were used following two passages. The tissues from the first-trimester pregnancy were immediately placed into cold DMEM, transported to the laboratory within $1 \mathrm{~h}$ following surgery, and washed with Hank's balanced salt solution for isolation of DSCs and decidual immune cells (DICs). The
DSCs and DICs were isolated as previously described (20), and the purity of DSCs was approximately 90\%. DSCs were used following two passages.

Isolation and culture of $d N K$ cells. $\mathrm{dNK}$ cells were isolated using a magnetic activated cell sorting human NK cell negative selection isolation kit (Miltenyi Biotec, Bergisch Gladbach, Germany) in accordance with the manufacturer's protocol. The collected $\mathrm{dNK}$ cells were incubated with fluorescein-isothiocyanate (FITC)-conjugated anti-CD56 (cat. no. 562794; 1:50; BD BioSciences, Franklin Lakes, NJ, USA) and allophycocyanin (APC)-conjugated anti-CD16 (cat. no. 17-0168-41; 1:50; eBioscience; Thermo Fisher Scientific, Inc.) monoclonal antibodies in a final volume of $100 \mu \mathrm{l}$ flow staining buffer for $30 \mathrm{~min}$ at $4^{\circ} \mathrm{C}$. Then, the purity of dNK cells was evaluated by FACScan flow cytometry (BD Biosciences, Franklin Lakes, NJ, USA), and data were analyzed by Flow Jo software (version 8.7, FlowJo LLC, Ashland, OR, USA).

ESC culture experiments. ESCs ( $2 \times 10^{5}$ cells per well) from six different patients were seeded in 12 -well plates and cultured at $37^{\circ} \mathrm{C}$ in a humidified atmosphere incubator containing $5 \% \mathrm{CO}_{2}$ and, following attachment to the wall, the medium was replaced with phenol red-free DMEM supplemented with $2 \%$ dextran-coated charcoal-treated fetal bovine serum (FBS; Hyclone; GE Healthcare Life Sciences, Logan, UT, USA). Decidualization of ESCs was induced with 8-br-cAMP (0.5 mM; Sigma-Aldrich; Merck KGaA, Darmstadt, Germany) and MPA (1 $\mu \mathrm{M}$; Sigma Aldrich; Merck KGaA), whereas the controls were treated with medium only. Next, to explore the role of IL-25 in decidualization, ESCs were treated with medium, cAMP+MPA and cAMP+MPA+recombinant human (rh) IL-25 respectively for 2, 4, 6 and 8 days. ESCs were also pre-treated with anti-IL-25 or anti-IL-17RB neutralizing antibody for $1 \mathrm{~h}$, and then stimulated with cAMP+MPA for decidualization. The working concentrations of recombinant human (rh) IL-25, anti-human IL-25 neutralizing antibody (anti-IL-25), and anti-IL-17RB (R\&D Systems, Inc., Minneapolis, MN, USA) were $1 \mathrm{ng} / \mathrm{ml}, 1 \mu \mathrm{g} / \mathrm{ml}$, and $1 \mu \mathrm{g} / \mathrm{ml}$, respectively. The media were replaced every 2 days.

Co-culture of dNK cells with ESCs. ESCs ( $2 \times 10^{5}$ cells per well) from six different patients were seeded in 12-well plates. Following pre-treatment with $0.5 \mathrm{mM} 8$-br-cAMP and $1 \mu \mathrm{M}$ MPA for 3 days, the supernatant was removed, and ESCs were co-cultured with dNK cells, induced NK cells (pretreated with 8-br-cAMP plus MPA for 3 days), and 8-br-cAMP plus MPA respectively, whereas the controls were treated with medium only. The supernatant was collected 3 days later to detect the concentration of PRL. In order to explore whether co-culture of dNK cells with ESCs affected the expression of IL-25, ESCs ( $1 \times 10^{5}$ cells per well) from six different patients were seeded in 24-well plates overnight. Then, $\mathrm{dNK}$ cells $\left(1 \times 10^{5}\right.$ cells per well) from six different patients were co-cultured with ESCs for $48 \mathrm{~h}$.

Enzyme-linked immunosorbent assay (ELISA). ESCs $\left(2 \times 10^{5}\right.$ cells per well) were seeded in 12 -well plates. Following culture in conditioned media for 2, 4, 6, and 8 days (D2, D4, D6, and D8, respectively), the culture supernatants 
A

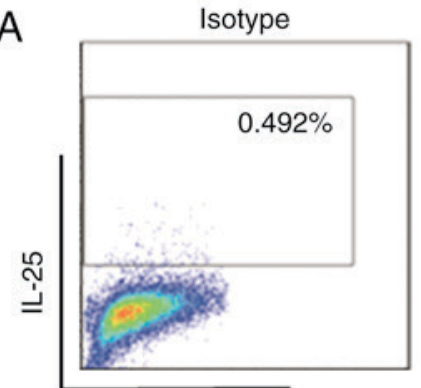

FSC

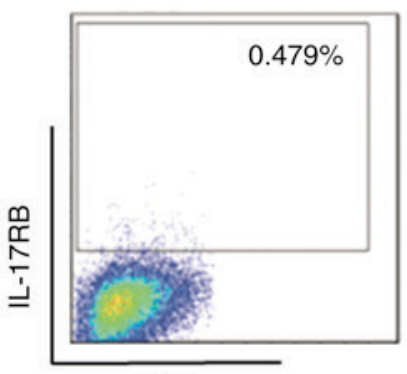

FSC
B

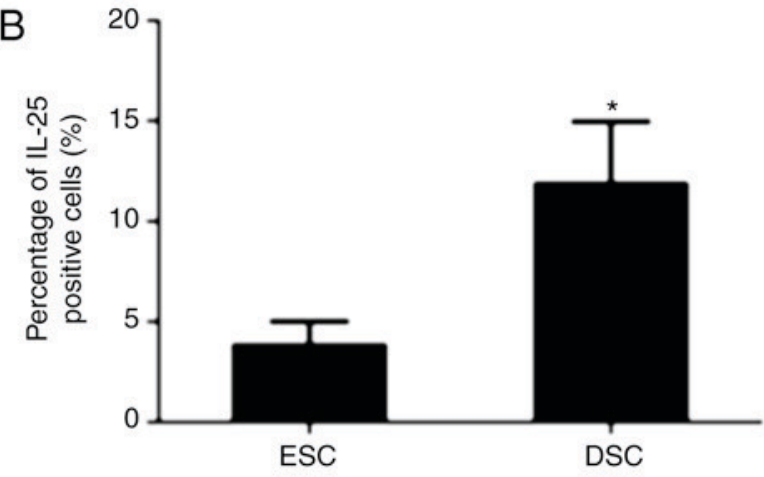

ESC
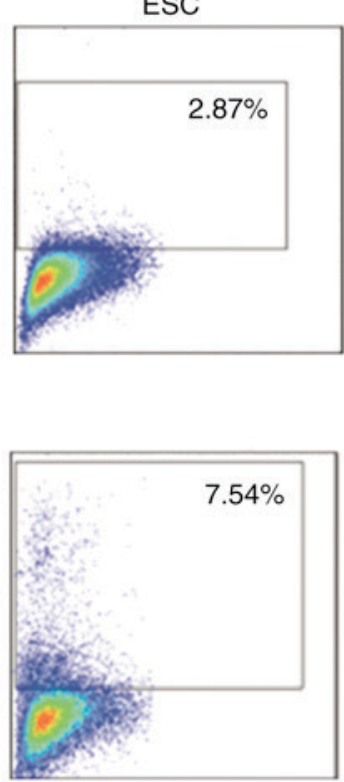

DSC
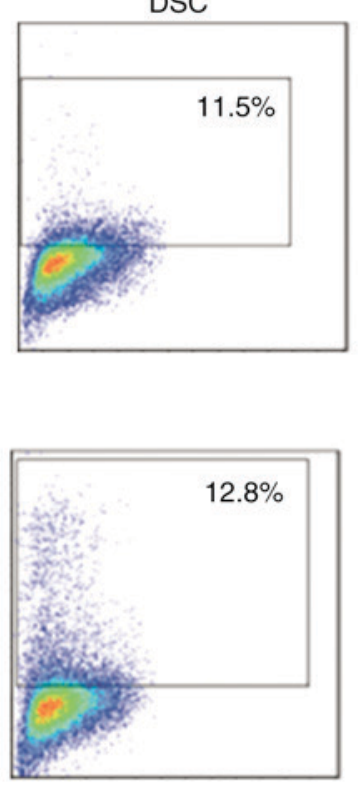

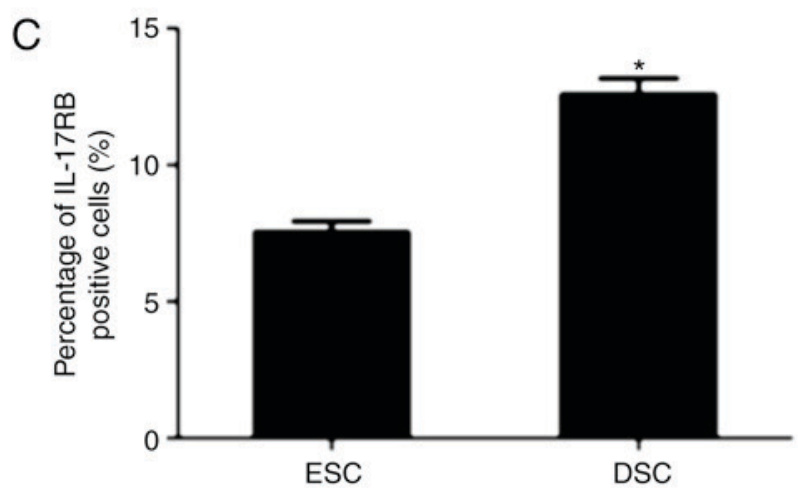

Figure 1. Expression of IL-25/IL-17RB in ESCs and DSCs. (A) Human primary ESCs ( $\mathrm{n}=6$ ) and DSCs ( $\mathrm{n}=6$ ) were isolated from normal healthy volunteers. The expression of IL-25/IL-17RB in ESCs and DSCs was detected by flow cytometry. Quantification of (B) IL-25 and (C) IL-17RB. DSCs expressed an increased level of IL-25 and IL-17RB compared with ESCs. Data are presented as mean \pm standard deviation. ${ }^{*} \mathrm{P}<0.05$ vs. ESCs. IL, interleukin; ESCs, endometrial stromal cells; DSCs, decidual stromal cells.

were harvested at $12,000 \mathrm{x}$ g for $10 \mathrm{~min}$ at $4^{\circ} \mathrm{C}$, and stored at $-80^{\circ} \mathrm{C}$. The concentration of PRL was detected by ELISA according to the manufacturer's protocol (KA0217; Alpha Diagnostic International Inc., San Antonio, TX, USA). dNK cells $\left(5 \times 10^{5}\right.$ cells per well) were seeded in 24 -well plates for 24, 48, 72 and $96 \mathrm{~h}$; the culture supernatants were harvested, centrifuged to remove cellular debris, and stored at $-80^{\circ} \mathrm{C}$. The concentration of IL-25 was detected by ELISA according to the manufacturer's protocol (Assay Biotech Company Inc., Sunnyvale, CA, USA).

Western blot analysis. ESCs ( $5 \times 10^{5}$ cells per well) were seeded in six-well plates. Following culture in conditioned media for 2 , 4, 6, and 8 days, the ESCs were lysed in radioimmunoprecipitation assay lysis buffer (Beyotime Institute of Biotechnology, Shanghai, China) containing $1 \%$ proteinase inhibitors (Beyotime Institute of Biotechnology). The protein concentration was detected by bicinchoninic acid assay (Beyotime Institute of Biotechnology). Each sample (25 $\mu \mathrm{g})$ was loaded on a $10 \%$ sodium dodecyl sulfate-polyacrylamide gel for electrophoresis, then the proteins were transferred onto a polyvinyl difluoride membrane (EMD Millipore, Billerica, MA, USA), followed by incubation of the membrane in blocking buffer containing 5\% non-fat dry milk for $1 \mathrm{~h}$ at room temperature. Next, the membrane was incubated overnight at $4^{\circ} \mathrm{C}$ with the following primary antibodies in blocking buffer: Polyclonal anti-human-IGFBP-1 (ab4249; 1:1,000; Abcam, Cambridge, MA, USA); monoclonal anti-human-actin (AF0003; 1:1,000; Beyotime Institute of Biotechnology). Then, following a 10 min wash, 3 times, the membrane was incubated with horseradish peroxidase-conjugated anti-rabbit or anti-mouse IgG secondary antibodies (NA931, NA934; 1:5,000; GE Healthcare, Chicago, IL, USA) for $1 \mathrm{~h}$ at room temperature. Blots were exposed via using the Amersham ECL detection kit (GE Healthcare). The results were scanned and densitometrically analyzed via ImageJ software (version 1.6.0, National Institutes of Health, Bethesda, MD, USA).

Flow cytometry. DSCs, ESCs, and dNK cells were collected separately and washed with phosphate buffered saline. Following blocking with $10 \%$ FBS $(1: 10)$ at room temperature, the recovered cells were mixed 

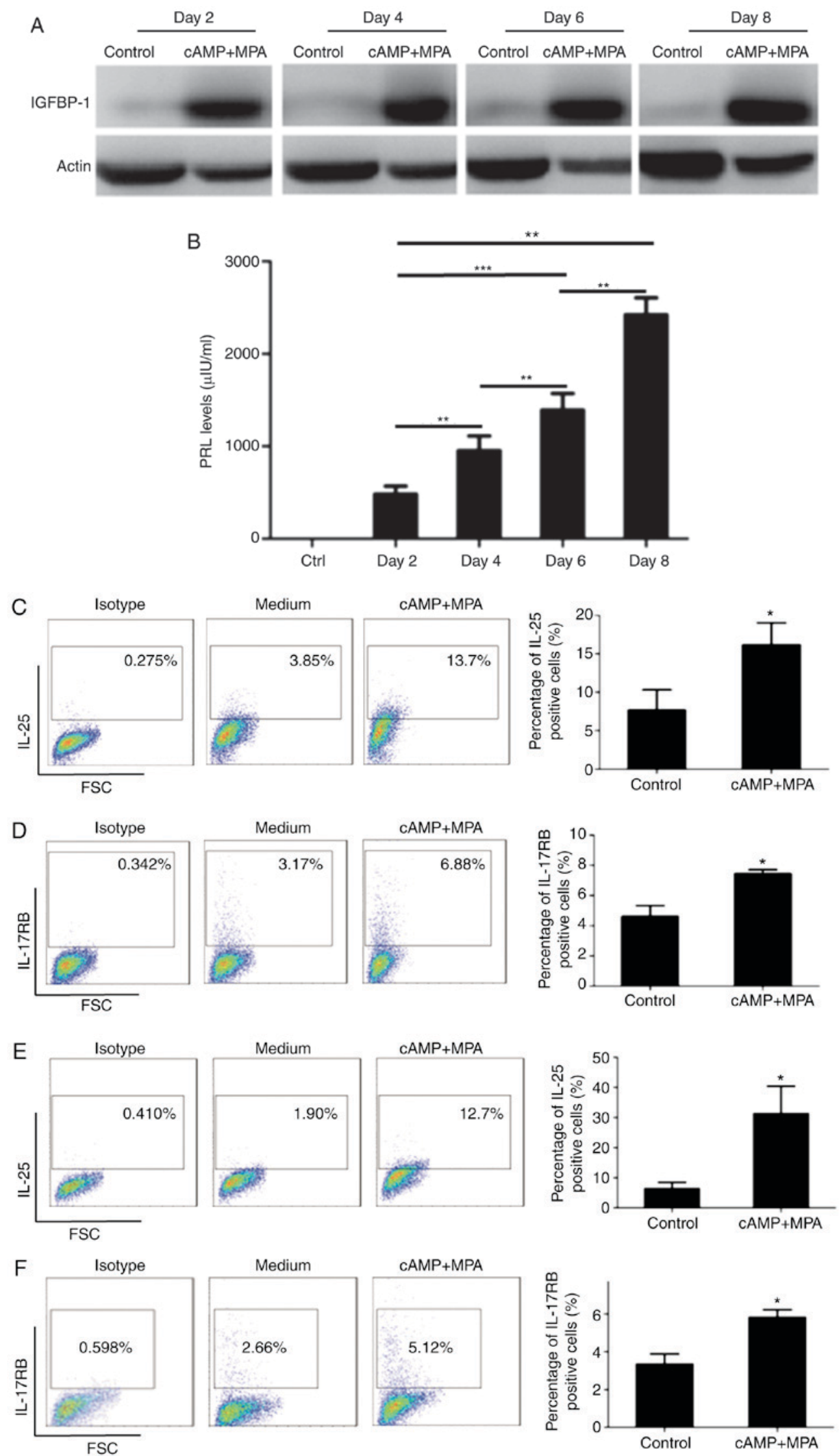

Figure 2. Decidualization increases the expression of IL-25/IL-17RB in ESCs. The establishment of an in vitro decidualization model. ESCs (n=6) were treated with $0.5 \mathrm{mM}$ 8-br-cAMP and $1 \mu \mathrm{M}$ MPA for 2, 4, 6 and 8 days (D2, D4, D6, and D8, respectively). (A) Expression level of IGFBP-1 was detected by western blot analysis, and (B) PRL level in the cell culture supernatant was detected by ELISA. ${ }^{* *} \mathrm{P}<0.01,{ }^{* * * *} \mathrm{P}<0.001$. Following in vitro decidualization, the expression of IL-25/IL-17RB in ESCs was detected. Following treatment with 8-br-cAMP and MPA, (C) IL-25 and (D) IL-17RB expression levels were detected at 2 days and (E) IL-25 and (F) IL-17RB levels detected again at 6 days via flow cytometry. ${ }^{*}<0.05$ vs. medium control. Data are presented as the mean \pm standard deviation. IL, interleukin; ESCs, endometrial stromal cells; 8-br-cAMP 8-bromoadenosine 3',5'-cyclic monophosphate sodium salt; MPA, 6 $\alpha$-methyl17 $\alpha$-acet oxyprogesterone; IGFBP-1, insulin growth factor binding protein 1; PRL, prolactin. 

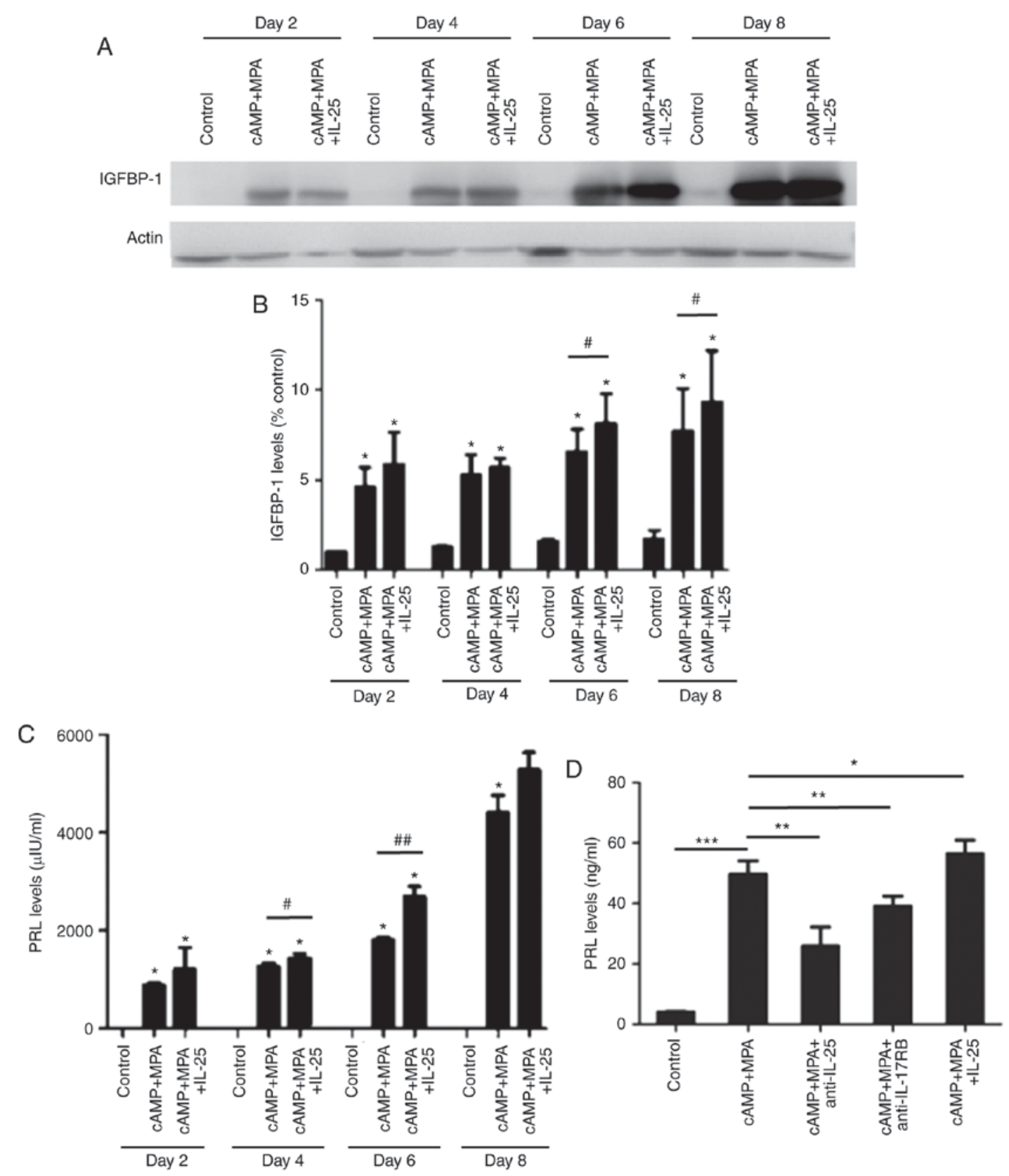

Figure 3. IL-25 promotes the decidualization of ESCs. Following treatment with medium, 8-br-cAMP plus MPA or 8-br-cAMP+MPA+recombinant human IL-25 for 2, 4, 6, and 8 days, the expression levels of IGFBP-1 and PRL was detected by western blot analysis and ELISA, respectively. (A) Representative western blot of IGFBP-1 in ESCs (n=6). (B) Densitometric quantification of IGFBP-1 in ESCs. ${ }^{*} \mathrm{P}<0.05$ vs. control group, " $\mathrm{P}<0.05$ vs. 8-br-cAMP plus MPA group. (C) The level of PRL secreted by ESCs $(\mathrm{n}=6)$ in the culture supernatant. ${ }^{*} \mathrm{P}<0.05$ vs. control group, ${ }^{~} \mathrm{P}<0.05,{ }^{\# \#} \mathrm{P}<0.01$ vs. 8-br-cAMP plus MPA group. (D) Following treatment with $1 \mu \mathrm{g} / \mathrm{ml}$ anti-IL-25 or anti-IL-17RB $1 \mathrm{~h}$ prior to the 8 -br-cAMP plus MPA treatment, the PRL level in the supernatant was detected by ELISA after a 6 day period. ${ }^{*} \mathrm{P}<0.05,{ }^{* *} \mathrm{P}<0.01$ vs. 8 -br-cAMP plus MPA group; ${ }^{* * *} \mathrm{P}<0.001$ vs. control group. Data are presented as the mean \pm standard deviation. IL, interleukin; ESCs, endometrial stromal cells; 8-br-cAMP 8-bromoadenosine 3',5'-cyclic monophosphate sodium salt; MPA, $6 \alpha$-methyl17 $\alpha$ -acetoxyprogesterone; IGFBP-1, insulin growth factor binding protein 1; PRL, prolactin; anti-IL, anti-human neutralizing antibody.

with APC-conjugated anti-IL-17RB [or phycoerythrin (PE)-conjugated anti-IL-17RB; FAB1207A or FAB1207P; 1:50; R\&D Systems, Inc.] in the dark for $45 \mathrm{~min}$ at room temperature, washed with phosphate buffered saline to remove unbound antibody. Then, following fixation and permeabilization (Fixation/Permeabilization Solution kit; 554714; $250 \mu \mathrm{l} /$ tube, BD Biosciences) for $30 \mathrm{~min}$ at room temperature, the cells were incubated with $\mathrm{PE}$-conjugated anti-IL-25 (or APC-conjugated anti-IL-25; 1:50, catalog. no. IC1258P or IC1258A, R\&D Systems, Inc.) for another $1 \mathrm{~h}$ at room temperature. The isotype control was used as a negative control. Following incubation, the cells were washed and analyzed immediately using a flow cytometer (FACS Beckman; BD BioSciences), and data were analyzed by Flow Jo software (version 8.7, FlowJo LLC).

Statistical analysis. The data were analyzed using the least significant difference test following one-way analysis of variance, or a Student's t-test. SPSS software, version 17.0 (SPSS, Inc., Chicago, IL, USA) was used to analyze data. All values are presented as the mean \pm standard deviation. $\mathrm{P}<0.05$ was considered to indicate a statistically significant difference. 

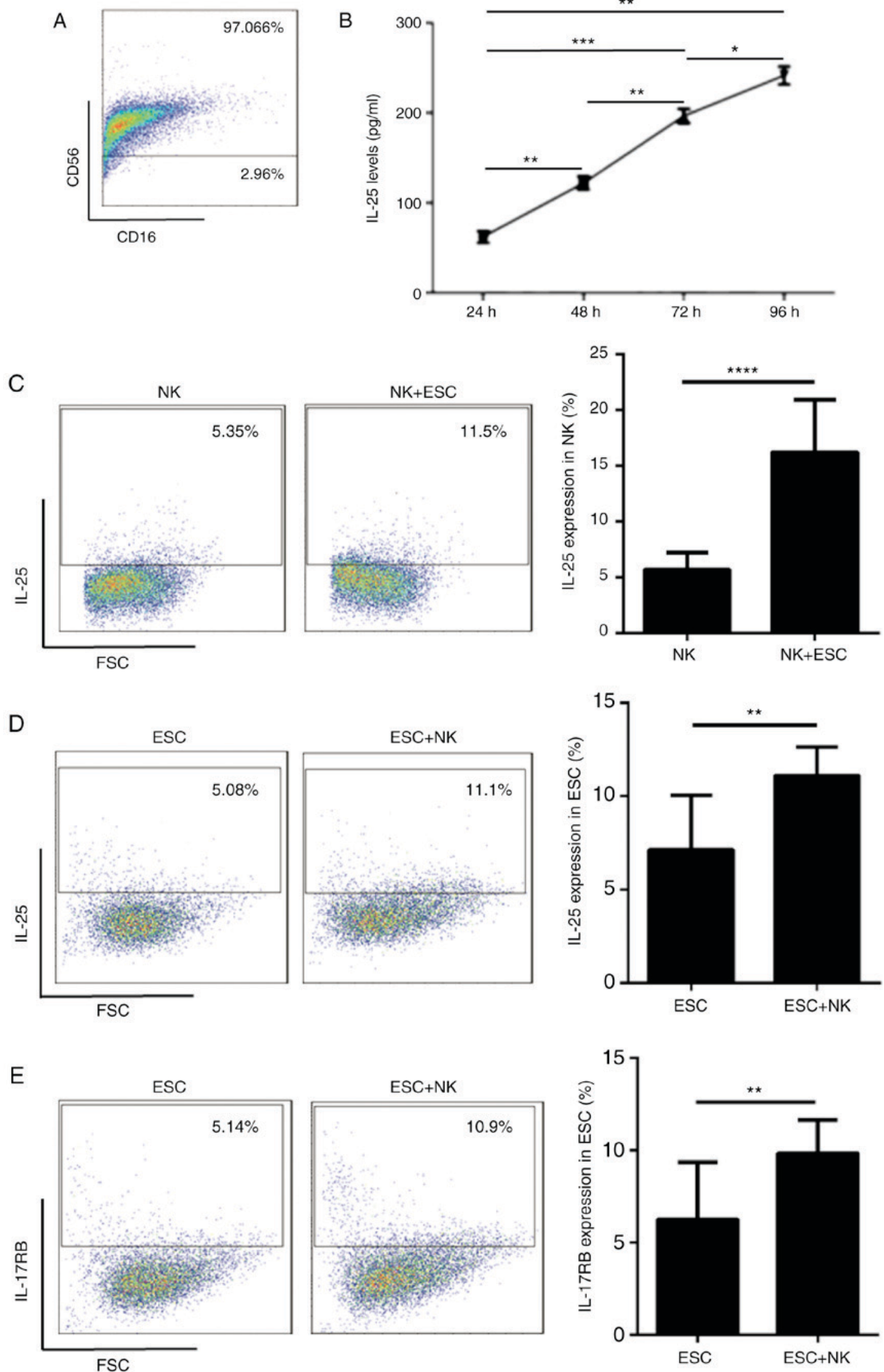

Figure 4. dNK cells and ESCs secrete IL-25, the level of which further increases following co-culture. dNK cells (n=6) secreted IL-25, the expression of which was increased following co-culture with ESCs. (A) Primary dNK cells were isolated from normal healthy volunteers, and the purity of dNK cells was evaluated by flow cytometry. (B) IL-25 secreted by dNK cells was detected by ELISA following $24,48,72$ and $96 \mathrm{~h} .{ }^{*} \mathrm{P}<0.05,{ }^{* * *} \mathrm{P}<0.01,{ }^{* * * *} \mathrm{P}<0.001$. (C) Expression of IL-25 in dNK cells alone was detected by flow cytometry, and then again following co-culture with ESCs for $48 \mathrm{~h}$. ${ }^{* * * * *} \mathrm{P}<0.0001$ vs. dNK cells-only group. The expression of (D) IL-25 and (E) IL-17RB in ESCs $(\mathrm{n}=6)$ alone, was detected by flow cytometry, and then again following co-culture with dNK cells for $48 \mathrm{~h}$. ${ }^{* *} \mathrm{P}<0.01$ vs. ESCs-only group. Data are presented as the mean \pm standard deviation. IL, interleukin; ESCs, endometrial stromal cells; dNK, decidual natural killer; $\mathrm{CD}$, cluster of differentiation. 


\section{Results}

Expression of IL-25 and IL-17RB in DSCs is increased compared with ESCs. Flow cytometry was used to investigate the expression level of IL-25 and IL-17RB in ESCs and compare it with DSCs. As presented in Fig. 1A-C, the percentage of IL-25- and IL-17RB-positive ESCs was decreased compared with DSCs, which indicated that the expression of IL-25 and IL-17RB in ESCs was increased following decidualization. Therefore, it was hypothesized that IL-25 may be involved in the decidualization of ESCs.

Decidualization upregulates the expression of IL-25/IL-17RB in ESCs. Next, to investigate the effect of decidualization on the expression of IL-25 and IL-17RB in ESCs, the present study induced the decidualization of ESCs in vitro using 8-br-cAMP and MPA. Following treatment with 8-br-cAMP and MPA for 2, 4, 6 and 8 days, the expression of IGFBP-1 and PRL in ESCs was detected by western blot analysis and ELISA, respectively (Fig. 2). As presented in Fig. 2A and B, the expression of IGFBP-1 increased following induction, compared with control group (Fig. 2A). The levels of PRL in the cell culture supernatant were also elevated in a time-dependent manner during the decidualization process (Fig. 2B). The results indicated that the establishment of an in vitro decidualization model was successful. Following treating ESCs with 8-br-cAMP plus MPA for 2 and 6 days, the expression levels of IL-25 and IL-17RB in ESCs were analyzed by flow cytometry, and it was demonstrated that the percentage of IL-25-positive ESCs (Fig. 2C and E) and IL-17RB-positive ESCs (Fig. 2D and F) was increased compared with the control group at 2 or 6 days. Therefore, these findings demonstrated that the expression of IL-25 and IL-17RB in ESCs was increased following decidualization in vitro, which was consistent with the results presented in Fig. 1.

IL-25 promotes the decidualization of ESCs in vitro. To explore the role of IL-25 in the process of decidualization, the present study induced the decidualization of ESCs in vitro by adding rhIL-25 to the conditioned media for 2, 4, 6 and 8 days. Then, the expression levels of IGFBP-1 and PRL were detected by western blot analysis and ELISA, respectively, which revealed that rhIL-25 further enhanced the expression of IGFBP-1 (Fig. 3A and B), in addition to the expression of PRL (Fig. 3C). However, when blocking IL-25 with anti-25 or anti-IL-17RB, the increase in PRL expression level was partially abrogated (Fig. 3D). Based on these findings, it was hypothesized that IL-25 promoted the decidualization of ESCs.

ESCs and dNK cells express $I L-25$. To investigate whether dNK cells secrete IL-25 at the maternal-fetal interface, the present study isolated primary dNK cells and using flow cytometry, identified their purity, which was $>95 \%$ (Fig. 4A). Then, dNK cells were cultured in vitro for $24,48,72$, and $96 \mathrm{~h}$, and the ELISA result indicated that dNK cells secreted IL-25 in a time-dependent manner (Fig. 4B). In addition, dNK cells were co-cultured with ESCs for $48 \mathrm{~h}$ to detect the expression of IL-25/IL-17RB. Data presented in Fig. 4C-E indicated that the expression of IL-25 in dNK cells (Fig. 4C) and the expression of IL-25/IL-17RB (Fig. 4D-E) in ESCs were increased

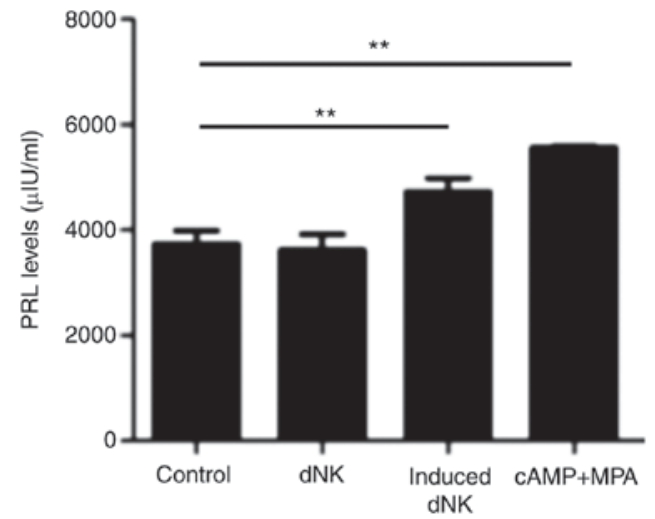

Figure 5. dNK cells promote decidualization. Following pretreatment with 8-br-cAMP plus MPA for 3 days, endometrial stromal cells were co-cultured with dNK cells and induced NK cells (pretreated with 8-br-cAMP plus MPA for 3 days) for another 3 days and the PRL level in the supernatant was detected by ELISA. ${ }^{* *} \mathrm{P}<0.01$ vs. control group. Data are presented as the mean \pm standard deviation. $\mathrm{dNK}$, decidual natural killer; 8-br-cAMP 8-bromoadenosine 3',5'-cyclic monophosphate sodium salt; MPA, $6 \alpha$-methyl17 $\alpha$-acetoxyprogesterone; PRL, prolactin.

following co-culture. Therefore, these results suggested that the dNK cells and ESCs secreted IL-25, the level of which was further increased following co-culture.

dNK cells promote the decidualization of ESCs. Next, the present study co-cultured ESCs (pretreated with 8-br-cAMP plus MPA for 3 days) with dNK cells, induced dNK cells (pretreated with 8-br-cAMP plus MPA for 3 days), and 8-br-cAMP plus MPA for another 3 days. The ELISA results of the PRL detection revealed that dNK cells alone did not induce the decidualization of ESCs, however, induced dNK cells promoted the process significantly (Fig. 5).

In conclusion, the results of the present study demonstrated that in normal pregnancy, ovarian hormones induce the decidualization of ESCs, resulting in the increased expression of IL-25/IL-17RB in ESCs (Fig. 6). The crosstalk between ESCs and dNK cells may further promote the decidualization in vitro by upregulating IL-25, thus contributing to the embryo implantation and successful pregnancy.

\section{Discussion}

It has previously been demonstrated that endometrial development and blastocyst implantation are critical for the initiation of pregnancy. The decidualization of uterine endometrium is one of the cornerstones of embryo implantation and maintenance of pregnancy. Decidualization, which usually occurs in the late secretory phase of the menstrual cycle, is defined as the proliferation and differentiation of ESCs under certain conditions. With fertilization, embryo implantation and trophoblastic invasion, the process of decidualization expands from the uterine spiral artery to the entire endometrium in a wavelike manner (21). In humans and mice, the level of decidualization is consistent with the level of trophoblastic invasion, regulating embryo implantation, placenta formation, and maintenance of normal pregnancy accurately. However, failure of decidualization may influence trophoblastic invasion, leading to infertility, recurrent 


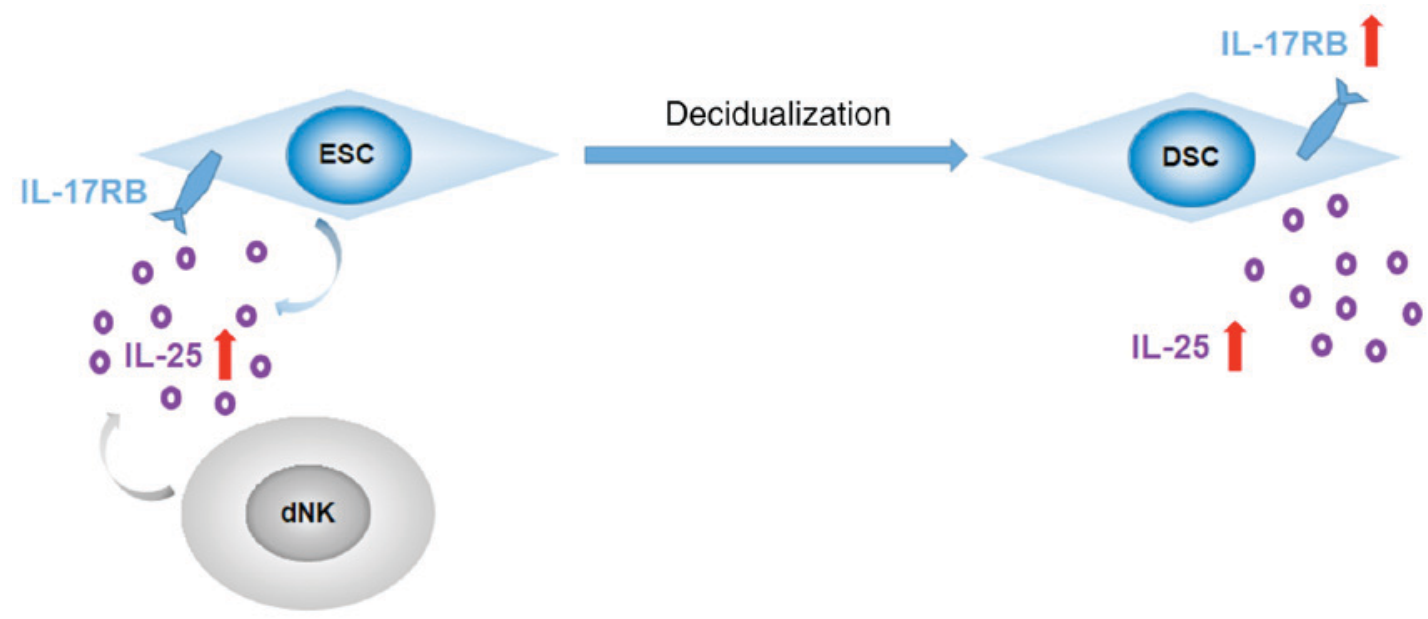

Figure 6. Process of decidualization in ESCs. ESCs undergo decidualization in the presence of ovarian hormones, leading to the increased expression of IL-25/IL-17RB. IL-25 secreted by ESCs and dNK cells further promotes the decidualization. IL, interleukin; ESCs, endometrial stromal cells; dNK, decidual natural killer.

miscarriage, pre-eclampsia, intra-uterine growth restriction, preterm birth and other diseases (22).

Currently, PRL and IGFBP-1 are widely used as symbols of decidualization (23-27). PRL, a polypeptide protein hormone secreted by the anterior lobe of the pituitary gland, is expressed in the anterior pituitary gland cells, skin fibroblasts, and DSCs (28). In addition to promoting latex secretion, PRL regulates multiple reproductive functions. Deficiency of PRL or its receptor in mice leads to embryo loss, degradation of fertilized eggs in the fallopian tube, and estrous cycle disorders (29). Furthermore, PRL inhibits the expression of IL-6 and $20 \alpha$-hydroxysteroid dehydrogenase, which are detrimental to pregnancy (30). IGFBP-1, localized to predecidualized stromal cells during the late secretory phase of endometrium and to DSCs in pregnant endometrium $(31,32)$, has been revealed to inhibit trophoblast invasion (33) and stimulate cell migration and adhesion (34). The combination of 8-br-cAMP and MPA mediates the maximal efficiency of decidualization (12), therefore the present study used cAMP together with MPA to induce the decidualization, which proved successful.

Based on the author's previous findings, it was speculated that IL-25 has an effect on the differentiation of ESCs into DSCs. The expression of IL-25/IL-17RB in ESCs and DSCs was investigated, and it was demonstrated that the expression level of IL-25/IL-17RB in DSCs was increased compared with ESCs. In addition, following decidualization in vitro, the expression of IL-25/IL-17RB in ESCs was significantly increased. Therefore, ESCs may express IL-25/IL-17RB, and the expression level is increased following decidualization. Then, by using rh-IL-25, it was revealed that IL-25 combined with 8-br-cAMP and MPA promoted the process more efficiently compared with 8-br-cAMP and MPA alone. Conversely, when blocking IL-25 with anti-IL-25 or anti-IL-17RB, the role of 8-br-cAMP and MPA in stimulating decidualization was partially weakened. Therefore, it was concluded that IL-25 promoted decidualization and that upregulation of IL-17RB in ESCs during decidualization increases the sensitivity of IL-25, which further facilitates the process. In addition, the AKT and JNK signaling pathways are involved in decidualization $(35,36)$, and the authors previously demonstrated that
IL-25 activates AKT and JNK signals in DSCs. Therefore, IL-25 may promote the decidualization via the AKT and JNK signaling pathways. cAMP, a secondary messenger, has been demonstrated to activate its downstream protein kinase A (PKA) (37), cAMP-gated ion channels (38), and exchange proteins activated by cAMP (39), as well as popeye domain containing proteins (40). It has been revealed that vasoactive intestinal peptide inhibits lipopolysaccharide-mediated IL-17A expression via the PKA and protein kinase C (PKC) pathways (41). Therefore, the PKA and PKC pathways may contribute to the increase of IL-25 expression induced by cAMP and MPA, which requires further verification.

dNK cells, most of which are CD56 $6^{\text {bright }} \mathrm{CD}^{-}$, account for $\sim 70 \%$ of the immune cell population in the decidua basalis (42). Previous studies have reported that decidualization is associated with recruitment of NK cells, which are low in proliferative phase endometrium, and increase in the mid-luteal and late secretory phases of the menstrual cycle $(43,44)$. These NK cells are favorable to embryo implantation and decidualization (45). Therefore, the present study explored the role of $\mathrm{dNK}$ cells in decidualization. As previously stated, dNK cells alone did not induce decidualization, whereas pretreatment of dNK cells with cAMP and MPA exhibited the opposite effect. dNK cells have been reported to promote decidualization in two ways: Promoting blood vessel formation (46) or secreting cytokines (47). In humans, dNK cells generate various angiogenic growth factors, including vascular endothelial growth factor, angiopoietin- 1,2 , transforming growth factor- $\beta$ (48), and placental growth factor $(49,50)$, however in mice, dNK cells secrete interferon- $\gamma$ for spiral artery remodeling (51). dNK cells may promote the decidualization by secreting IL-25, accompanied by the aforementioned factors, however, the specific mechanism requires further investigation.

\section{Acknowledgements}

The present study was supported by the National Basic Research Program of China (grant no. 2015CB943300), the National Natural Science Foundation of China (grant no. 81671460), the Program for Shanghai leaders, the Program of Shanghai 
Outstanding Academic Leader (grant no. 15XD1500900) and the Foundations from Shanghai Science and Technology Committee (grant no. 134119a4300) to Li-Ping Jin, and Foundation of Shanghai First Maternity and Infant Hospital, Tongji University School of Medicine (grant no. 2016A17) to Xiao-hui Wang.

\section{References}

1. Zhang $\mathrm{S}$, Lin $\mathrm{H}$, Kong $\mathrm{S}$, Wang $\mathrm{S}$, Wang $\mathrm{H}$, Wang $\mathrm{H}$ and Armant DR: Physiological and molecular determinants of embryo implantation. Mol Aspects Med 34: 939-980, 2013.

2. Tang B, Guller S and Gurpide E: Mechanisms of human endometrial stromal cell decidualization. Ann N Y Acad Sci 734: 19-25, 1994.

3. Salamonsen LA, Dimitriadis E, Jones RL and Nie G: Complex regulation of decidualization: A role for cytokines and proteases-a review. Placenta 24 (Suppl A): S76-S85, 2003.

4. Maruyama T and Yoshimura Y: Molecular and cellular mechanisms for differentiation and regeneration of the uterine endometrium. Endocr J 55: 795-810, 2008.

5. Matsumoto K, Yamauchi N, Watanabe R, Oozono S, Kubota K, Nishimura K, Wood C, Soh T, Kizaki K and Hattori MA: In vitro decidualization of rat endometrial stromal cells. Cell Tissue Res 335: 575-583, 2009.

6. Salamonsen LA, Nie G, Hannan NJ and Dimitriadis E: Society for reproductive biology Founders' Lecture 2009. Preparing fertile soil: The importance of endometrial receptivity. Reprod Fertil Dev 21: 923-934, 2009.

7. Dimitriadis E, White CA, Jones RL and Salamonsen LA: Cytokines, chemokines and growth factors in endometrium related to implantation. Hum Reprod Update 11: 613-630, 2005.

8. Koot YE, Boomsma CM, Eijkemans MJ, Lentjes EG and Macklon NS: Recurrent pre-clinical pregnancy loss is unlikely to be a 'cause' of unexplained infertility. Hum Reprod 26 : 2636-2641, 2011.

9. Aplin JD: Developmental cell biology of human villous trophoblast: Current research problems. Int J Dev Biol 54: 323-329, 2010

10. Knöfler M: Critical growth factors and signalling pathways controlling human trophoblast invasion. Int J Dev Biol 54 269-280, 2010.

11. Popovici RM, Kao LC and Giudice LC: Discovery of new inducible genes in in vitro decidualized human endometrial stromal cells using microarray technology. Endocrinology 141: $3510-3513,2000$

12. Gellersen B and Brosens J: Cyclic AMP and progesterone receptor cross-talk in human endometrium: A decidualizing affair. J Endocrinol 178: 357-372, 2003.

13. Brosens JJ, Hayashi N and White JO: Progesterone receptor regulates decidual prolactin expression in differentiating human endometrial stromal cells. Endocrinology 140: 4809-4820, 1999.

14. Fort MM, Cheung J, Yen D, Li J, Zurawski SM, Lo S, Menon S, Clifford T, Hunte B, Lesley R, et al: IL-25 induces IL-4, IL-5, and IL-13 and Th2-associated pathologies in vivo. Immunity 15: 985-995, 2001.

15. Corrigan CJ, Wang W, Meng Q, Fang C, Wu H, Reay V, Lv Z, Fan Y, An Y, Wang YH, et al: T-helper cell type 2 (Th2) memory T cell-potentiating cytokine IL-25 has the potential to promote angiogenesis in asthma. Proc Natl Acad Sci USA 108: 1579-1584, 2011.

16. Wang W, Fan YQ, Lv Z, Yao XJ, Wang W, Huang KW, Meng Q, Fang CL, Lee TH, Corrigan CJ, et al: Interleukin-25 promotes basic fibroblast growth factor expression by human endothelial cells through interaction with IL-17RB, but not IL-17RA. Clin Exp Allergy 42: 1604-1614, 2012.

17. Giannubilo SR, Landi B, Pozzi V, Sartini D, Cecati M, Stortoni P, Corradetti A, Saccucci F, Tranquilli AL and Emanuelli M: The involvement of inflammatory cytokines in the pathogenesis of recurrent miscarriage. Cytokine 58: 50-56, 2012.

18. Wang Y, Zhang Y, Li MQ, Fan DX, Wang XH, Li DJ and Jin LP: Interleukin-25 induced by human chorionic gonadotropin promotes the proliferation of decidual stromal cells by activation of JNK and AKT signal pathways. Fertil Steril 102: 257-263, 2014

19. Mei J, Xie XX, Li MQ, Wei CY, Jin LP, Li DJ and Zhu XY: Indoleamine 2,3-dioxygenase-1 (IDO1) in human endometrial stromal cells induces macrophage tolerance through interleukin-33 in the progression of endometriosis. Int J Clin Exp Pathol 7: 2743-2757, 2014.
20. Fu Q, Tao Y,Piao H, Du MR and Li DJ: Trophoblasts and decidual stromal cells regulate decidual NK cell functions via interaction between collagen and LAIR-1. Am J Reprod Immunol 71: 368-378, 2014.

21. Ramathal CY, Bagchi IC, Taylor RN and Bagchi MK: Endometrial decidualization: Of mice and men. Semin Reprod Med 28: 17-26, 2010.

22. Salker M, Teklenburg G, Molokhia M, Lavery S, Trew G, Aojanepong $\mathrm{T}$, Mardon $\mathrm{HJ}$, Lokugamage AU, Rai R, Landles C, et al: Natural selection of human embryos: Impaired decidualization of endometrium disables embryo-maternal interactions and causes recurrent pregnancy loss. PLoS One 5: e10287, 2010.

23. Telgmann R and Gellersen B: Marker genes of decidualization: Activation of the decidual prolactin gene. Hum Reprod Update 4: 472-479, 1998

24. Pohnke Y, Kempf R and Gellersen B: CCAAT/enhancer-binding proteins are mediators in the protein kinase A-dependent activation of the decidual prolactin promoter. J Biol Chem 274: 24808-24818, 1999.

25. Kim JJ, Taylor HS, Akbas GE, Foucher I, Trembleau A, Jaffe RC, Fazleabas AT and Unterman TG: Regulation of insulin-like growth factor binding protein-1 promoter activity by FKHR and HOXA10 in primate endometrial cells. Biol Reprod 68: 24-30, 2003.

26. Grinius L, Kessler C, Schroeder J and Handwerger S: Forkhead transcription factor FOXO1A is critical for induction of human decidualization. J Endocrinol 189: 179-187, 2006.

27. Lynch VJ, Brayer K, Gellersen B and Wagner GP: HoxA-11 and FOXO1A cooperate to regulate decidual prolactin expression: Towards inferring the core transcriptional regulators of decidual genes. PLoS One 4: e6845, 2009.

28. Gellersen B, Kempf R, Telgmann R and DiMattia GE: Nonpituitary human prolactin gene transcription is independent of Pit-1 and differentially controlled in lymphocytes and in endometrial stroma. Mol Endocrinol 8: 356-373, 1994.

29. Binart N, Helloco C, Ormandy CJ, Barra J, Clément-Lacroix P, Baran N and Kelly PA: Rescue of preimplantatory egg development and embryo implantation in prolactin receptor-deficient mice after progesterone administration. Endocrinology 141: 2691-2697, 2000

30. Bao L, Tessier C, Prigent-Tessier A, Li F, Buzzio OL, Callegari EA, Horseman ND and Gibori G: Decidual prolactin silences the expression of genes detrimental to pregnancy. Endocrinology 148: 2326-2334, 2007.

31. Julkunen M, Koistinen R, Suikkari AM, Seppälä $M$ and Jänne OA: Identification by hybridization histochemistry of human endometrial cells expressing mRNAs encoding a uterine beta-lactoglobulin homologue and insulin-like growth factor-binding protein-1. Mol Endocrinol 4: 700-707, 1990.

32. Rutanen EM, Partanen S and Pekonen F: Decidual transformation of human extrauterine mesenchymal cells is associated with the appearance of insulin-like growth factor-binding protein-1. J Clin Endocrinol Metab 72: 27-31, 1991.

33. Irwin JC and Giudice LC: Insulin-like growth factor binding protein-1 binds to placental cytotrophoblast alpha5betal integrin and inhibits cytotrophoblast invasion into decidualized endometrial stromal cultures. Growth Horm IGF Res 8: 21-31, 1998.

34. Jones JI, Gockerman A, Busby WH Jr, Wright G and Clemmons DR: Insulin-like growth factor-binding protein 1 stimulates cell migration and binds to the alpha 5 beta 1 integrin by means of its Arg-Gly-Asp sequence. Proc Natl Acad Sci USA 90: 10553-10557, 1993.

35. Leitao B, Jones MC, Fusi L, Higham J, Lee Y, Takano M, Goto T, Christian M, Lam EW and Brosens JJ: Silencing of the JNK pathway maintains progesterone receptor activity in decidualizing human endometrial stromal cells exposed to oxidative stress signals. FASEB J 24: 1541-1551, 2010.

36. Fabi F and Asselin E: Expression, activation, and role of AKT isoforms in the uterus. Reproduction 148: R85-R95, 2014

37. Meinkoth JL, Alberts AS, Went W, Fantozzi D, Taylor SS, Hagiwara M, Montminy M and Feramisco JR: Signal transduction through the cAMP-dependent protein kinase. Mol Cell Biochem 127-128: 179-186, 1993.

38. Kaupp UB and Seifert R: Cyclic nucleotide-gated ion channels. Physiol Rev 82: 769-824, 2002.

39. Bos JL: Epac proteins: Multi-purpose cAMP targets. Trends Biochem Sci 31: 680-686, 2006. 
40. Simrick S, Schindler RF, Poon KL and Brand T: Popeye domain-containing proteins and stress-mediated modulation of cardiac pacemaking. Trends Cardiovasc Med 23: 257-263, 2013.

41. Ran WZ, Dong L, Tang CY, Zhou Y, Sun GY, Liu T, Liu YP and Guan CX: Vasoactive intestinal peptide suppresses macrophage-mediated inflammation by downregulating interleukin-17A expression via PKA- and PKC-dependent pathways. Int J Exp Pathol 96: 269-275, 2015.

42. Rabot M, Tabiasco J, Polgar B, Aguerre-Girr M, Berrebi A, Bensussan A, Strbo N, Rukavina D and Le Bouteiller P: HLA class I/NK cell receptor interaction in early human decidua basalis: Possible functional consequences. Chem Immunol Allergy 89: 72-83, 2005.

43. Bulmer JN, Morrison L, Longfellow M, Ritson A and Pace D: Granulated lymphocytes in human endometrium: Histochemical and immunehistochemical studies. Hum Reprod 6: 791-798, 1991.

44. Loke YW, King A and Burrows TD: Decidua in human implantation. Hum Reprod (Suppl 2): S14-S21, 1995.

45. Lukassen HG, Joosten I, van Cranenbroek B, van Lierop MJ, Bulten J, Braat DD and van der Meer A: Hormonal stimulation for IVF treatment positively affects the CD56bright/CD56dim NK cell ratio of the endometrium during the window of implantation. Mol Hum Reprod 10: 513-520, 2004.

46. Leonard S, Murrant C, Tayade C, van den Heuvel M, Watering R and Croy BA: Mechanisms regulating immune cell contributions to spiral artery modification-facts and hypotheses-a review. Placenta 27 (Suppl A): S40-S46, 2006.
47. Jabrane-Ferrat $\mathrm{N}$ and Siewiera J: The up side of decidual natural killer cells: New developments in immunology of pregnancy. Immunology 141: 490-497, 2014

48. Hanna J, Goldman-Wohl D, Hamani Y, Avraham I, Greenfield C, Natanson-Yaron S, Prus D, Cohen-Daniel L, Arnon TI, Manaster I, et al: Decidual NK cells regulate key developmental processes at the human fetal-maternal interface. Nat Med 12: 1065-1074, 2006.

49. Levine RJ, Maynard SE, Qian C, Lim KH, England LJ, Yu KF, Schisterman EF, Thadhani R, Sachs BP, Epstein FH, et al: Circulating angiogenic factors and the risk of preeclampsia. $\mathrm{N}$ Engl J Med 350: 672-683, 2004.

50. Chappell LC, Duckworth S, Seed PT, Griffin M, Myers J, Mackillop L, Simpson N, Waugh J, Anumba D, Kenny LC, et al: Diagnostic accuracy of placental growth factor in women with suspected preeclampsia: A prospective multicenter study. Circulation 128: 2121-2131, 2013.

51. Ashkar AA, Di Santo JP and Croy BA: Interferon gamma contributesto initiation of uterine vascular modification, decidual integrity, and uterine natural killer cell maturation during normal murine pregnancy. J Exp Med 192: 259-270, 2000.

This work is licensed under a Creative Commons Attribution-NonCommercial-NoDerivatives 4.0 International (CC BY-NC-ND 4.0) License. 